\title{
SEMI-HOMOMORPHISMS OF RINGS
}

\author{
S.A. HuQ
}

In this note, following Kaplanasky's study of semi-automorphisms of rings and Herstein's study of semi-homomorphisms of groups, we present a general study of semi-homomorphisms of rings.

\section{0 . Introduction}

Perhaps the notion of a semi-homomorphism was conceived as a common generalization of both the notion of a homomorphism and an anti-homomorphism. The study was begun, for semi-automorphisms only, by Anchochea [1] and Kaplanasky [4]. Anchochea, studied semi-automorphisms of quaternion algebras and division algebras and proved that if $A$ is a simple algebra of characteristic $\neq 2$, then a semi-automorphism of $A$ is either an automorphism or an anti-automorphism. This was extended by Kaplanasky [4] to simple algebras over any field. Later on Hua [3] proved that a semiautomorphism of any skewfield is either an automorphism or an antiautomorphism. Herstein [2] proceeded later on to study semi-homomorphisms of groups. Here, following Herstein and Kaplanasky, we present the study of semi-homomorphisms of rings in general.

\section{Main Results}

DEFINITION 1. Let $R$ be a ring and $S$ a subset of $R$; we call $S$ a semi-subring, if for all $x, y \in S$

Received 18 August 1986. The author is grateful to ICTP, Trieste for a two weeks grant in the summer of 1985 where the idea of this paper was conceived and to Monash University for a visiting grant in 1986, when this paper was completed.

Copyright Clearance Centre, Inc. Serial-fee code: 0004-9729/87 $\$ A 2.00+0.00$ 
(i) $x+y+x \in S$.

(ii) $x y x \in S$.

Every subring is a semi-subring but the converse need not be true.

DEFINITION 2. A mapping $\phi: R \rightarrow S$ between two rings is called a semi-homomorphism, if for $a, b \in R$

(i) $\phi(a+b+a)=\phi(a)+\phi(b)+\phi(a)$ and

(ii) $\phi(a b a)=\phi(a) \phi(b) \phi(a)$

hold. Clearly any homomorphism or antihomomorphism is a semi-homomorphism but the converse need not be true; for example the constant function from $z_{2}$ to $z_{2}$ having value 1 , is a semi-endomorphism of $z_{2}$ but not an endomorphism.

The set $K=\{x \mid \phi(x)=0\}$ if it exists is called the kernel of the semi-homomorphism $\phi$. One notices that $K$ and $\phi(K)$ are then semisubrings of $R$ and $S$ respectively.

PROPOSITION 3. If $\phi: R \rightarrow R^{\prime}$ is a semi-homomorphism of mings, then for $a \in R$

$$
\phi(-a)=-\phi(a) .
$$

Proof. $\phi(a)=\phi[a+(-a)+a]=\phi(a)+\phi(-a)+\phi(a)$ from which our result follows.

LEMMA 4. A semi-homomorphism $\phi: R \rightarrow R^{\prime}$ of mings will be a homomorphism of the underlying additive groups if the characteristic of the codomain $R^{\prime} \neq 2$,

Proof. For $a, b \in R$

$$
\begin{aligned}
\phi(a+b)=\phi[(a+b)+[-(a+b)]+a+b] \\
=\phi[a+b+[-(a+b)]+b+a] . \\
=\phi(a)+\phi[b+[-(a+b)]+b]+\phi(a) . \\
=\phi(a)+\phi(b)+\phi[-(a+b)]+\phi(b)+\phi(a) . \\
=2 \phi(a)+2 \phi(b)-\phi(a+b) \text { by proposition } 1.3
\end{aligned}
$$

That is $\quad 2[\phi(a+b)-\phi(a)-\phi(b)]=0$.

$$
\text { Thus } \phi(a+b)=\phi(a)+\phi(b) \text { since char } R^{\prime} \neq 2 \text {. }
$$

A consequence of Lemma 4 is the corollary:

COROLLARY 5. For a semi-homomorphism $\phi: R \rightarrow R^{\prime}$ with char $R^{\prime} \neq 2$. we have $\phi(-n a)=-n \phi(a)$ for any integer $n$. 
PROPOSITION 6. If $\phi: R \rightarrow R^{\prime}$ is a semi-homomorphism of rings with identities $1, I^{\prime}$ respectively and if $I^{\prime} \epsilon \phi(R)$, then $[\phi(1)]^{2}=I^{\prime}$. If further $R^{\prime}$ is a nontrivial ring without zero divisors, then $\phi(1)=1^{\prime}$ or $-1^{\prime}$ (which are distinct when char $R^{\prime} \neq 2$ ).

Proof. If $1^{\prime}=\phi(r)$ for $r \in R$ then $\phi(x)=\phi(1) \phi(x) \phi(1)$ so $\phi(r)=[\phi(1)]^{2}$. Hence $[\phi(1)]^{2}=1^{\prime}$.

Now $[\phi(1)]^{2}-1^{\prime}=0 \Rightarrow\left[\phi(1)+1^{\prime}\right]\left[\phi(1)-1^{\prime}\right]=0$ from which the second part follows.

PROPOSITION 7. If $\phi: F \rightarrow F^{\prime}$ is a semi-homomorphism of fields, then the kernel of $\phi$ is either zero or a regular semi-subring, provided char $F^{\prime} \neq 2$.

Proof. One notices that if the char $F^{\prime}=2$, the kernel may fail to exist. For the definition of reqularity, we refer to [3]. Thus if $K \neq 0$, let $a \neq 0 \in K$, then $a^{-1}=a^{-1} a a^{-1} \in K$. Thus $a=a a^{-1} a$ for $a^{-1} \in K$.

PROPOSITION 8. For fields $F, F^{\prime}$ and a semi-homomorphism $\phi: F \rightarrow F^{\prime}$, if $a \neq 0 \in F$, does not belong to the kermel of $\phi$, then

$$
\phi\left(a^{-1}\right)=[\phi(a)]^{-1}
$$

Proof.

$$
\phi(a)=\phi\left(a a^{-1} a\right)=\phi(a) \phi\left(a^{-1}\right) \phi(a)
$$

Therefore

$$
\phi\left(a^{-1}\right)=[\phi(a)]^{-1} \text {. }
$$

PROPOSITION 9. If $\phi: F+F^{\prime}$ is not a nulz semi-homomorphism of fiezds, then

$$
\phi(1) \in \text { Centre of } \phi(F) \text {. }
$$

Proof. Since $\phi$ is not null $I \notin \operatorname{Ker} \phi$, if Ker $\phi$ exists. Therefore as $\phi(1)=[\phi(1)]^{-1}$ by proposition 8 ; that is $[\phi(1)]^{2}=1$ ' so

$$
\begin{aligned}
& \phi(x)=\phi(1) \phi(r) \phi(1), \\
& \phi(1) \phi(r)=\phi(r) \phi(1) .
\end{aligned}
$$

and

Now we ask when is a semi-homomorphism a homomorphism?

We present a sufficient condition in a special situation. 
THEOREM 10. A semi-monomorphism $\phi: R \rightarrow R^{\prime}$ of rings will be a monamorphism, if

(i) char $R^{\prime} \neq 2$,

(ii) $\phi(R)$ is a skew subfield of $R^{\prime}$ and

(iii) $\phi(2 y+y z)-2 \phi(y)=\phi(y z y)[\phi(y)]^{-1}$.

Proof. (i) By Lemma 4, (i) guarantees $\phi$ to be a homomorphism for the underlying additive groups. If either of $y, z=0$, then $\phi(y)$ and $\phi(z)=0$, or $\phi(z)=0$ and $\phi(y z)=0$, so in either case $\phi(y z)=\phi(y)(z)$.

When both $y, z \neq 0$, then $\phi(y)$ and $\phi(z)$ both $\neq 0$

so

$$
\phi(y z y)=\phi(y) \phi(z) \phi(y)
$$

implies

$$
\phi(y) \phi(z)=[\phi(y z y)][\phi(y)]^{-1} \text {. }
$$

Also

that is

$$
\phi(2 y+y z)=\phi(y+y z+y)=\phi(y)+\phi(y z)+\phi(y)
$$

Hence by (iii),

$$
\phi(2 y+y z)-2 \phi(y)=\phi(y z) \text {. }
$$

One notices that (iii) in Theorem 10 can be equivalently replaced by

(iii)'

$$
\phi(z y)=[\phi(y)]^{-1} \phi(y z y) \text {. }
$$

The open problem here is to find the necessary and sufficient condition for a semi-homomorphism to be a homomorphism.

THEOREM 11. For commutative ring $R$ and $R^{\prime}$ with identities, if $\phi: R \rightarrow R^{\prime}$ is on identity-preserving semi-homomorphism, and char $R^{\prime} \neq 2$, then $\phi$ is a homomorphism or an antihomomorphism.

Proof. We know by Lemma $4, \phi$ is an additive homomorphism.

Now

$$
\phi[(x+y) \cdot 1(x+y)]=\phi(x+y) \cdot 1^{\prime} \cdot \phi(x+y)
$$

that is $\phi[x \cdot x+x \cdot y+y \cdot x+y \cdot y]=\phi(x) \phi(x)+\phi(x) \phi(y)+\phi(y) \phi(x)+\phi(y \cdot y)$. Then using $\phi(x .1 . x)=\phi(x) .1^{\prime} . \phi(x)$ and since $\phi$ is additive we have,

that is

$$
\phi(x y)+\phi(y x)=\phi(x) \phi(y)+\phi(y) \phi(x)
$$

that is

$$
\begin{aligned}
2 \phi(x y) & =2 \phi(x) \phi(y) \\
\phi(x y) & =\phi(x) \phi(y) .
\end{aligned}
$$

A slight weakening of Hua's theorem now reads as:

THEOREM 12. If $\phi: K \rightarrow K^{\prime}$ is con identity preserving semimonomorphism of skew fields, then $\phi$ is either a monomorphism or an 
conti-monomorphism, provided char $K^{\prime} \neq 2$.

Proof. Applying Lemma 4, one proceeds to prove this result exactly as in Hua [3].

Remarks. (A) The set of semi-endomorphisms of an abelian group $A$ form a ring, the ring of semi-endomorphisms of the group $A$ which contains the ring of endomorphism as a subring, under the usual pointwise addition and composition of functions. Thus any ring $(R,+, \ldots)$ can be embedded into the ring of semi-endomorphism of $(R,+)$.

(B) The concept of a semi-homomorphism becomes more significant when both the additive and the multiplicative structures are not commutative, for example, near-ring [4]. This case if left for future study.

\section{References}

[1] G. Anchochea, "On semi-automorphisms of division algebras", Ann. of Math., 48 (1947), 147-153.

[2] I.N. Herstein, "Semi-homomorphisms of groups", Canad. J. Math. 20 (1968), 384-388.

[3] L.K. Hua, "On the automorphisms of a field", Proc. Nat. Acad. Sci. U.S.A., 35 (1949), 386-389.

[4] I. Kaplanasky, "Semi-automorphisms of Rings", Duke Math. J., 14 (1947), 521-525.

[5] N.H. CcCoy, Theory of rings, (Collier-Macmillan, 1968).

[6] G. Pilz, Neor-rings, (North Holland, 1977).

Department of Mathematics

Monash University, Clayton

Victoria 3168, Australia.

and

Faculty of Science

Sana'a University

YEMEM ARAB REPUBLIC. 